\title{
NONCROSSED PRODUCTS OF SMALL EXPONENT
}

\author{
DAVID J. SALTMAN ${ }^{1}$
}

\begin{abstract}
Using the generic division algebras, we construct new division algebras and prove the following. THEOREM. Let $m, n$ be positive integers, $m \mid n$, such that $m$ is divisible by every prime which divides $n$. If $k$ is any field, and there is a prime $p$ such that $p^{3} \mid m$, then there is a noncrossed product division $k$-algebra of exponent $m$ and degree $n$. If $k$ is a global field of characteristic $0, p$ is an odd prime, and $k$ does not contain a primitive pth root of 1 , we need only assume $p^{2} \mid m$.
\end{abstract}

In [Ro], Rowen showed that any division algebra of degree 8 and exponent 2 is a crossed product. This fact leads one to ask when one could construct noncrossed product division algebras of less than full exponent. Some such results are presented here. The reader should note that the noncrossed product division algebras so far constructed, the generic division algebras (e.g. [A, p. 417]), are of full exponent. We will use the generic noncrossed product algebras to construct others with the desired properties. In what follows, $k$ will always be a field and $k((x))$ the Laurent series field with coefficients in $k$.

We first require the following observation about the generic division algebra $U D(k, n, q)$ over $k$, in $q$ variables, of degree $n$; $q$ will always be $\geqslant 2$.

LEMMA 1. If $L / k$ is a finite extension of fields, $U D(k, n, q) \otimes_{k} L \cong$ $U D(L, n, q)$.

Proof. Let $k\left(x_{i j}^{m}\right)$ be the purely transcendental extension of $k$ with transcendence base $\left\{x_{i j}^{m} \mid 1 \leqslant i, j \leqslant n ; 1 \leqslant m \leqslant q\right\}$. Recall that $U D(k, n, q) \subseteq$ $M_{n}\left(k\left(x_{i j}^{m}\right)\right)$ is the subdivision ring generated by $k$ and the generic matrices

$$
X^{m}=\left(x_{i j}^{m}\right), \quad 1 \leqslant m \leqslant q .
$$

Embedding $M_{n}\left(k\left(x_{i j}^{m}\right)\right) \subseteq M_{n}\left(L\left(x_{i j}^{m}\right)\right)$ in the natural way, $U D(L, n, q)$ is generated as a division ring by $U D(k, n, q)$ and $L$. Since

$$
M_{n}\left(k\left(x_{i j}^{m}\right)\right) \otimes_{k} L \cong M_{n}\left(k\left(x_{i j}^{m}\right)\right) L=M_{n}\left(L\left(x_{i j}^{m}\right)\right),
$$

one can conclude that

$$
U D(k, n, q) \otimes_{k} L \cong U D(k, n, q) L \subseteq U D(L, n, q) .
$$

But $U D(k, n, q) L$ is a domain, finite-dimensional over the center of

Received by the editors June 24, 1977.

AMS (MOS) subject classifications (1970). Primary 16A40; Secondary 16A16.

Key words and phrases. Division algebra, crossed product.

'The author gratefully acknowledges support under NSF Grant MCS 76-06626. 
$U D(k, n, q)$, and thus is a division ring. Hence $U D(k, n, q) L=$ $U D(L, n, q)$. Q.E.D.

Now we record some well-known facts about division algebras $D$ finitedimensional and central over a complete discrete valuation field $L$ with residue class field $K$ (e.g. [Re, Chapter 3] or [Sc, Chapter 5]). $D$ has a ramification degree $e(D / L)$, which is also the maximum ramification degree of any $L$-subfield of $D . D$ has a residue class division algebra $\bar{D}$ and if $f=[\bar{D}: K]$ is the dimension of $\bar{D}$ over $K$, ef $=n^{2}=[D: L]$. For any $L$ subfield $F$ of $D$, the residue class field $\bar{F}$ is a subfield of $\bar{D}$. Finally, iff $e(D / L)=1$, and $\bar{D}$ has center $K$ it follows from, e.g. [Se, p. 195], that if $F / L$ splits $D, \bar{F} / K$ splits $\bar{D}$.

Suppose $A$ is a $k$ central simple algebra, and $L / k$ is a cyclic Galois extension of fields. Let $K=k((x)), F=L((x))$, and $\sigma$ be a generator of the Galois group $G(L / k)$. Define

$$
(A, L, x)=\left(A \otimes_{k} K\right) \otimes_{K}(F / K, \sigma, x),
$$

where $(F / K, \sigma, x)$ is the cyclic algebra as defined in, for example, [J, p. 82]. Then:

THEOREM 2. (a) If $M, L$ are linearly disjoint fields over $k$,

$$
(A, L, x) \otimes_{K} M((x)) \cong\left(A \otimes_{k} M, M L, x\right) .
$$

(b) If the exponent of $A$ in the Brauer group $\operatorname{Br}(k)$ is $n$, and $m=[L: k]$, then the exponent of $(A, L, x)$ is the least common multiple $[m, n]$.

(c) If $A \otimes_{k} L$ is a division algebra, so is $(A, L, x)$.

(d) Suppose $A \otimes_{k} L$ is a noncrossed product division algebra. Then $(A, L, x)$ is a noncrossed product.

Proof. (a) This is immediate from the definition.

(b) Let $k_{a}$ be the algebraic closure of $k$ and $\mathrm{Br}_{u}(k((x)))$ be the subgroup, of the Brauer group, of all elements split by an unramified extension of $k((x))$. It is immediate that the equivalence class $[(A, L, x)]$ is in $\mathrm{Br}_{u}(K)$. By, for example, [Sc, Chapter 5, §7], $\mathrm{Br}_{u}(K)=\operatorname{Br}(k) \oplus \chi(G)$. Here $G$ is $G\left(k_{a} / k\right)$ and $\chi(G)$ is the character group $\operatorname{Hom}(G, Q / Z)$. Looking at the proof, it is easy to see that $[(A, L, x)]$ corresponds to $[A] \oplus(f)$, where $f$ is the character mapping $G\left(k_{a} / L\right)$ to 1 and $\sigma$ to $1 / m$. Part (b) now follows easily.

(c) Extend $\sigma$ to an automorphism $\sigma^{\prime}$ on $A \otimes_{k} L=A^{\prime}$ by letting $\sigma^{\prime}$ act trivially on $A .(A, L, x)$ is just the twisted Laurent series division algebra $A^{\prime}\left(\left(x, \sigma^{\prime}\right)\right)($ e.g. $[\mathrm{J}$, p. 87]).

(d) Let $D=(A, L, x)$. Then $\bar{D}=A \otimes_{k} L$ and $e(D / L)=[L: k]$. Suppose $N \subseteq D$ is a Galois maximal subfield. By [E, p. 107], $\bar{N} / K$ is a normal extension. Since $N$ splits $D, N F$ splits $D$ and $(F / K, \sigma, x)$ so $N F$ splits $A \otimes_{k} K . A \otimes_{k} K$ is unramified. Since $F$ is unramified it can be shown that $\overline{N F}=\bar{N} L$. But $\bar{N} L$ splits $A$, and $\bar{N}$ is a subfield of $A \otimes_{k} L$, implying that $\bar{N} L$ is a maximal subfield of $\bar{D}=A \otimes_{k} L$. As $\bar{N} L / L$ is normal, it will suffice to prove the following lemma. 
LEMMA 3. If $D$ is a $k$ central division algebra with a normal maximal subfield $F$, then $D$ is a crossed product.

Proof. We will use the ideas and notation of [S1]. Since $F / k$ is normal, $F=L P$ where $L / k$ is Galois and $P / k$ is purely inseparable. Our argument will proceed by induction on $[P: k]$. Choose $P^{\prime} \subseteq P$ such that $p=$ char $k=$ $\left[P: P^{\prime}\right]$ and $P=P^{\prime}(\alpha)$ where $\alpha^{p}=a \in P^{\prime}$. Call $D^{*}$ the centralizer of $L P^{\prime}$ in $D$. Then $D^{*} \cong[b, a)$ for some $b \in L P^{\prime}$. By Lemma 6 of $[\mathbf{S 1}], D^{*} \cong\left[b^{\prime}, a^{\prime}\right)$ where $b^{\prime} \in P^{\prime}$. By Lemma 2 of $[\mathbf{S} 1],\left[b^{\prime}, a^{\prime}\right) \cong\left[\left(b^{\prime}\right)^{p}, a^{\prime}\right)$ and so we may assume $b^{\prime} \in k$. Thus there is a $\beta \in D^{*}$ such that $\beta^{p}-\beta=b^{\prime}$. Calling $F^{\prime}=L(\beta) P^{\prime}, F^{\prime}$ is a maximal subfield of $D$. We are done by induction.

Suppose $A$ is a $k$ central simple algebra, and $L_{1}, \ldots, L_{N}$ are linearly disjoint cyclic extensions of $k$. Define, recursively, $\mathscr{D}^{i}(A)$, by $\mathscr{Q}^{0}(A)=A$ and $\mathscr{Q}^{i}(A)=\left(\mathscr{Q}^{i-1}(A), L_{i}^{\prime}, x_{i}\right)$ where $L_{i}^{\prime}=L_{i}\left(\left(x_{1}, \ldots, x_{i-1}\right)\right)$. Theorem 2(a) and induction easily show that if $M / k$ is linearly disjoint from $L_{1}, \ldots, L_{i}$, and $K=k\left(\left(x_{1}, \ldots, x_{i}\right)\right)$ is the center of $\mathscr{Q D}^{i}(A)$, then

$$
\mathscr{Q}^{i}(A) \otimes_{K} M\left(\left(x_{1}, \ldots, x_{i}\right)\right) \cong \mathscr{Q}^{i}\left(A \otimes_{k} M\right) \text {. }
$$

Here $\mathscr{Q}^{i}\left(A \otimes_{k} M\right)$ is computed with respect to $L_{1} M / M, \ldots, L_{i} M / M$.

Let $p$ be a prime, and $k, r$ such that $D=U D\left(k, p^{r}, q\right)$ is a noncrossed product, necessarily of degree and exponent $p^{r}$. Assume $L_{1}^{*}, \ldots, L_{N}^{*}$ are linearly disjoint cyclic extensions of $k$. Call $C$ the center of $D$. Since $C \subseteq k\left(x_{i j}^{m}\right), k$ is algebraically closed in $C$. Thus $L_{i}=C \otimes_{k} L_{i}^{*}$ are linearly disjoint cyclic extensions of $C$ and $\left[L_{i}: C\right]=\left[L_{i}^{*}: k\right]$. Define $\mathscr{Q}^{i}(D)$ using the $L_{i}$ 's. Assume that for any field $L, k \subseteq L \subseteq L_{1}^{*} L_{2}^{*} \cdots L_{N}^{*}, U D\left(L, p^{r}, q\right)$ is a noncrossed product.

THEOREM 4. $\mathscr{Q}^{i}(D)$ is a noncrossed product division algebra. If $q_{j}=\left[L_{j}^{*}: k\right]$, then $\mathscr{D}^{i}(D)$ has degree $p^{r} q_{1} \cdots q_{i}$ and exponent the least common multiple of $p^{r}, q_{1}, \ldots, q_{i}$.

Proof. First of all we show, by induction, that $\mathscr{D}^{i}(D)$ is a noncrossed product division algebra. By Theorem 2 , it suffices to show

$$
\mathscr{D}^{i-1}(D) \otimes L_{i}\left(\left(x_{1}, \ldots, x_{i-1}\right)\right)
$$

is a noncrossed product division algebra. But by our above remark,

$$
\begin{aligned}
\mathscr{Q}^{i-1}(D) \otimes L_{i}\left(\left(x_{1}, \ldots, x_{i-1}\right)\right) & \cong \mathscr{Q}^{i-1}\left(D \otimes_{C} L_{i}\right) \\
\cong \mathbb{D}^{i-1}\left(D \otimes_{k} L_{i}^{*}\right) & =\mathscr{Q}^{i-1}\left(U D\left(L_{i}^{*}, p^{r}, q\right)\right) .
\end{aligned}
$$

We are done, after applying the induction hypothesis to $\mathscr{Q}^{i-1}$ and $U D\left(L_{i}^{*}, p^{r}, q\right)$.

That the degree of $\mathscr{Q}^{i}(D)$ is $p^{r} q_{1} \cdots q_{i}$ is immediate. As for the exponent, the result is clear using Theorem 2(b). Q.E.D.

We have developed the above machinery in order to prove the following theorem, the goal of this paper.

THEOREM 5. Let $m, n$ be two positive integers, $m \mid n$, such that $m$ is divisible by 
all the primes which divide $n$. If for some prime $p, p^{3} \mid m$, then there is a noncrossed product division $k$ algebra of exponent $m$ and degree $n$. If $k$ is $a$ global field of characteristic $0, p$ is an odd prime, and $k$ does not contain $a$ primitive pth root of 1 , we need only assume $p^{2} \mid m$.

Proof. Let $p^{r}$ be the highest power of $p$ dividing $m$. Given the assumptions, by [A, p. 417]; [FS, p. 190] and [S2], $D=U D\left(k, p^{r}, q\right)$ is a noncrossed product. It is easy to find $q_{1}, \ldots, q_{N}$ such that $p^{r} q_{1} \cdots q_{N}=n$ and the least common multiple of $p^{r}, q_{1}, \ldots, q_{N}$ is $m$. Enlarge $k$, if necessary, so that $k$ has linearly disjoint cyclic extensions $L_{1}^{*}, \ldots, L_{N}^{*}$ satisfying $\left[L_{i}^{*}: k\right]=q_{i}$. If $r \geqslant 3$, then $U D\left(L, p^{r}, q\right)$ is a noncrossed product and considering $\mathscr{D}^{N}(D)$, we are done in this case.

If $k$ is a global field of characteristic 0 containing no primitive $p$ th roots of 1 , it will suffice to show that one can choose cyclic extensions $L_{i}^{*} / k$ such that $\left[L_{i}^{*}: k\right]=q_{i}$ and such that $L_{1}^{*} \cdots L_{N}^{*}$ does not contain a primitive $p$ th root of 1 . Let $\mathcal{P}$ be a prime of $k$ such that the completion $k_{\odot}$ does not contain a primitive $p$ th root of 1 . Using the Grunwald-Wang Theorem (e.g. [AT, p. 105]) one can choose $L_{i}^{*} / k$ linearly disjoint of degree $q_{i}$, such that the local degree of $L_{i}^{*} / k$ at $\mathscr{P}$ is 1 . It follows that the local degree of $L_{1}^{*} \cdots L_{N}^{*} / k$ is 1 at $\mathscr{P}$, so $L_{1}^{*} \cdots L_{N}^{*}$ has no primitive $p$ th root of 1. Q.E.D.

\section{BIBLIOGRAPHY}

[A] S. A. Amitsur, On central division algebras, Israel J. Math. 12 (1972), 408-420.

[AT] E. Artin and J. Tate, Class field theory, Benjamin, New York, 1974.

[E] O. Endler, Valuation theory, Springer-Verlag, Berlin, 1972.

[FS] B. Fein and M. Schacher, Galois groups and division algebras, J. Algebra 38 (1976), 182-191.

[J] N. Jacobson, PI algebras, An introduction, Springer-Verlag, Berlin, 1975.

[Re] I. Reiner, Maximal orders, Academic Press, New York, 1975.

[Ro] L. Rowen, Central simple algebras (preprint).

[S1] D. Saltman, Splittings of cyclic p algebras, Proc. Amer. Math. Soc. 62 (1977), 223-228.

[S2] , Noncrossed product algebras and Galois p extensions, J. Algebra (to appear).

[Sc] O. F. C. Schilling, The theory of valuations, Math. Surveys, vol. 4, Amer. Math. Soc., Providence, R. I., 1950.

[Se] J. Serre, Corps locaux, Hermann, Paris, 1962.

Department of Mathematics, University of Chicago, Chicago, Illinois 60637 